\title{
A Complex of Incompressible Surfaces for handlebodies and the Mapping Class Group.
}

\author{
Charalampos Charitos $\dagger$, Ioannis Papadoperakis $\dagger$ \\ and Georgios Tsapogas $\ddagger$ \\ $\dagger$ Agricultural University of Athens \\ and $\ddagger$ University of the Aegean
}

March 24, 2022

\begin{abstract}
For a genus $g$ handlebody $H_{g}$ a simplicial complex, with vertices being isotopy classes of certain incompressible surfaces in $H_{g}$, is constructed and several properties are established. In particular, this complex naturally contains, as a subcomplex, the complex of curves of the surface $\partial H_{g}$. As in the classical theory, the group of automorphisms of this complex is identified with the mapping class group of the handlebody. 1
\end{abstract}

\section{Definitions and statements of results}

For a compact surface $F$, the complex of curves $\mathcal{C}(F)$, introduced by Harvey in [6], has vertices the isotopy classes of essential, non-boundary-parallel simple closed curves in $F$. A collection of vertices spans a simplex exactly when any two of them may be represented by disjoint curves, or equivalently when there is a collection of representatives for all of them, any two of which are disjoint. Analogously, for a 3 -manifold $M$, the disk complex $\mathcal{D}(M)$ is defined by using the proper isotopy classes of compressing disks for $M$ as the vertices. It was introduced in [12], where it was used in the study of mapping class groups of 3 -manifolds. In [11], it was shown to be a quasi-convex subset of $\mathcal{C}(\partial M)$.

By $H_{g}$ we denote the 3 -dimensional handlebody of genus $g \geq 2$. Recall that a compact connected surface $S \subset H_{g}$ with boundary is properly embedded if $S \cap \partial H_{g}=\partial S$ and $S$ is transverse to $\partial H_{g}$. A compressing disk for $S$ is a properly embedded disk $D$ such that $\partial D$ is essential in $S$. A properly embedded surface $S \subset H_{g}$ is incompressible if there are no compressing disks for $S$. Recall also that a

${ }^{1} 2010$ Mathematics Subject Classification: 57N10, 57N35 
map $F: S \times[0,1] \rightarrow H_{g}$ is a proper isotopy if for all $t \in[0,1],\left.F\right|_{S \times\{t\}}$ is a proper embedding. In this case we will be saying that $F(S \times\{0\})$ and $F(S \times\{1\})$ are properly isotopic in $H_{g}$ and we will use the symbol $\simeq$ to indicate isotopy in all cases (curves, surfaces etc).

Definition 1 Let $\mathcal{I}\left(H_{g}\right)$ be the simplicial complex whose vertices are the proper isotopy classes of compressing disks for $\partial H_{g}$ and of properly imbedded boundaryparallel incompressible annuli and pairs of pants in $H_{g}$. For a vertex $[S]$ which is not a class of compressing disks, it is also required that $S$ is isotopic to a surface $\bar{S}$ embedded in $\partial H_{g}$ via an isotopy

$$
F: S \times[0,1] \rightarrow H_{g}
$$

with $F(S \times\{0\})=S, F(S \times\{1\})=\bar{S}$ and $F$ being proper when restricted to $[0,1)$. A collection of vertices spans a simplex in $\mathcal{I}\left(H_{g}\right)$ when any two of them may be represented by disjoint surfaces in $H_{g}$.

Note that the class of properly embedded incompressible surfaces in $H_{g}$ is very rich. For example, it contains surfaces of arbitrarily high genus (see [13], [3]) which are not included as vertices in the complex $\mathcal{I}\left(H_{g}\right)$ defined above. Also observe that there exist properly embedded annuli and pairs of pants which are not isotopic to a surface entirely contained in $\partial H_{g}$. The isotopy classes of such surfaces are also excluded from the vertex set of $\mathcal{I}\left(H_{g}\right)$.

Note that we may regard $\mathcal{D}\left(H_{g}\right)$ as a subcomplex of $\mathcal{I}\left(H_{g}\right)$ or, by taking boundaries of the representative disks, of $\mathcal{C}\left(\partial H_{g}\right)$. Note also that the vertices of $\mathcal{I}\left(H_{g}\right)$ represented by annuli correspond exactly to the vertices of $\mathcal{C}\left(\partial H_{g}\right)$ represented by curves that are essential in $\partial H_{g}$ but are not meridian boundaries. We define the complex of annuli $\mathcal{A}\left(H_{g}\right)$ to be the subcomplex of $\mathcal{I}\left(H_{g}\right)$ spanned by these vertices. Together, the vertices of $\mathcal{D}\left(H_{g}\right) \cup \mathcal{A}\left(H_{g}\right)$ span a copy of $\mathcal{C}\left(\partial H_{g}\right)$ in $\mathcal{I}\left(H_{g}\right)$, and we regard $\mathcal{C}\left(\partial H_{g}\right)$ as a subcomplex of $\mathcal{I}\left(H_{g}\right)$.

Our goal is to show that for a handlebody $H_{g}$ of genus $g \geq 2$ the automorphisms of the complex $\mathcal{I}\left(H_{g}\right)$ are all geometric, that is, they are induced by homeomorphisms of $H_{g}$. This can be rephrased by saying that the map

$$
A: \mathcal{M C G}\left(H_{g}\right) \rightarrow \operatorname{Aut}\left(\mathcal{I}\left(H_{g}\right)\right)
$$

is an onto map, where $A u t\left(\mathcal{I}\left(H_{g}\right)\right)$ is the group of automorphisms of the complex $\mathcal{I}\left(H_{g}\right)$ and $\mathcal{M C G}\left(H_{g}\right)$ is the (extended) mapping class group of $H_{g}$, i.e. the group of isotopy classes of self-homeomorphisms of $H_{g}$. Moreover, we will show (see Theorem 7 below) that the map $A$ is $1-1$ except when $H_{g}$ is the handlebody of genus 2 in which case a $\mathbb{Z}_{2}$ kernel is present generated by the hyper-elliptic involution.

For the proof of this result we perform a close examination of links of vertices in $\mathcal{I}\left(H_{g}\right)$. This examination establishes that an automorphism $f$ of $\mathcal{I}\left(H_{g}\right)$ must 


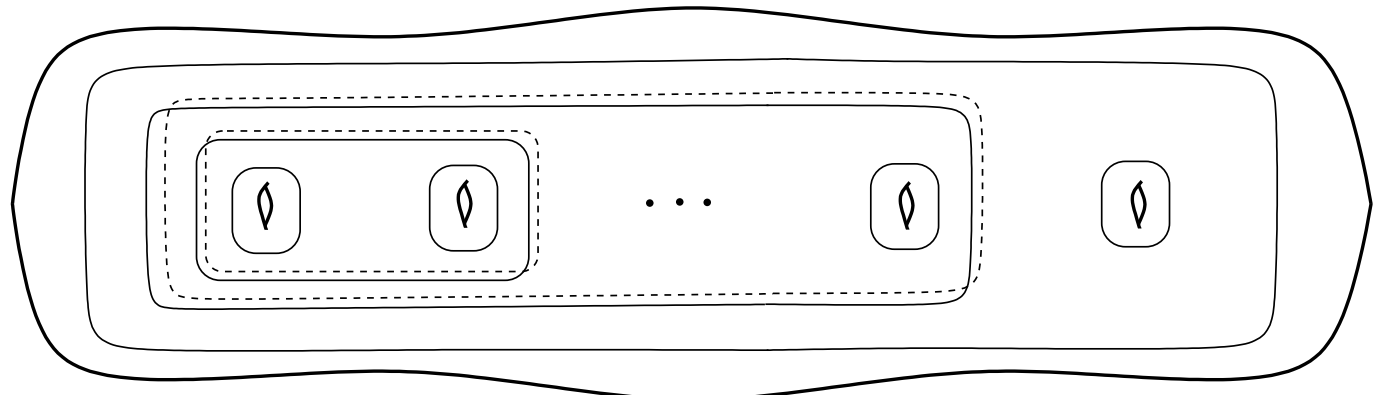

Figure 1: Pants decomposition for $H_{g}$ consisting of non-separating, non-meridian curves, $g \geq 3$.

map each vertex $v$ in $\mathcal{I}\left(H_{g}\right)$ to a vertex $f(v)$ consisting of surfaces of the same topological type as those in $v$. In particular, $f$ induces an automorphism of the subcomplex $\mathcal{C}\left(\partial H_{g}\right)$ which permits the use of the corresponding result for surfaces (see [7], [9]).

It is a well known result that for genus $\geq 2$ the complex of curves $\mathcal{C}\left(\partial H_{g}\right)$ is a $\delta$-hyperbolic metric space in the sense of Gromov (see [10], [2]). In the last section we deduce that the complex $\mathcal{I}(M)$ is itself a $\delta$-hyperbolic metric space in the sense of Gromov. Moreover, it follows that $\operatorname{Aut}\left(\mathcal{I}\left(H_{g}\right)\right)$ does not contain parabolic elements and the hyperbolic isometries of $\mathcal{I}(M)$ correspond to pseudo-Anosov elements of $\mathcal{M C G}\left(H_{g}\right)$.

In a recent preprint of M. Korkmaz and S. Schleimer (see [8]), it was shown, in a more general context, that $\mathcal{M C G}\left(H_{g}\right)$ and $A u t\left(\mathcal{D}\left(H_{g}\right)\right)$ are isomorphic. Apart from this isomorphism, our motivation for constructing the copmplex $\mathcal{I}\left(H_{g}\right)$ is the study of the mapping class group of a Heegaard splitting in a 3 -manifold $M$. This group (originally defined for $\mathbb{S}^{3}$ and often called the Goeritz mapping class group) consists of the isotopy classes of orientation preserving homeomorphisms of $M$ that preserve the Heegaard splitting. The mapping class group of a Heegaard splitting is known to be finitely presented (see [1], [4, [14]) only for $M=\mathbb{S}^{3}$ and for a genus 2 Heegaard splitting. We aim to examine the corresponding open questions for $M=\mathbb{S}^{3}$ and Heegaard splittings of genus $\geq 3$ as well as for certain classes of hyperbolic 3-manifolds. For these purposes, the complex $\mathcal{I}\left(H_{g}\right)$ is a suitable building block for defining a complex encoding the complexity of the Goeritz mapping class group, because $\mathcal{I}\left(H_{g}\right)$ contains a copy of the curve complex of the boundary surface $\partial H_{g}$.

\subsection{Notation and terminology}

A 3-dimensional handlebody $H_{g}$ of genus $g$ can be represented as the union of a handle of index 0 (i.e. a 3 -ball) with $g$ handles of index 1 (i.e. $g$ copies of 


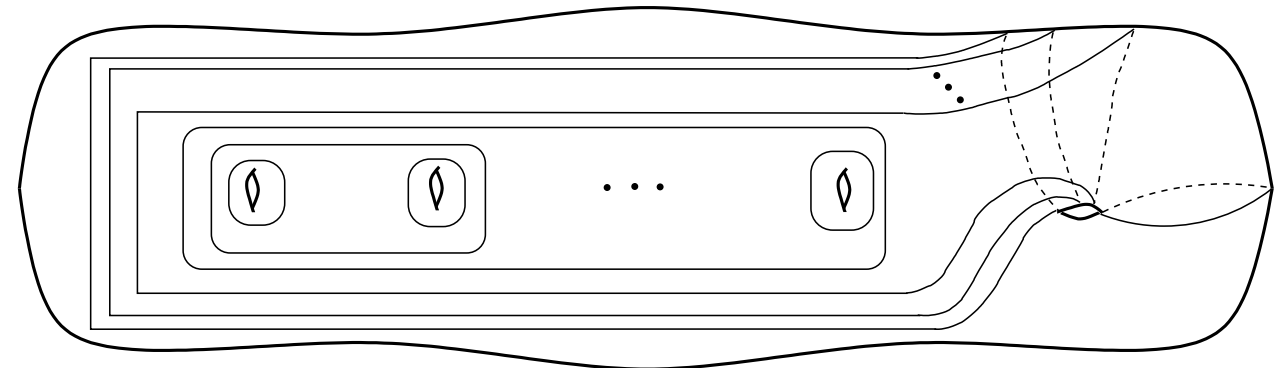

Figure 2: Pants decomposition for $H_{g}$ consisting of a single non-separating meridian curve, and $3 g-4$ non-meridian curves, $g \geq 3$.

$\left.D^{2} \times[0,1]\right)$

For an essential simple closed curve $\alpha$ in $\partial H_{g}$ we will be writing $[\alpha]$ for its isotopy class and the corresponding vertex in $\mathcal{C}\left(\partial H_{g}\right)$. We will be writing $\left[S_{\alpha}\right]$ for the corresponding vertex in $\mathcal{A}\left(H_{g}\right)$ where $S_{\alpha}$ is the annulus corresponding to the curve $\alpha$, provided that $\alpha$ is not a meridian boundary. We will be saying that $\left[S_{\alpha}\right]$ is an annular vertex. If $\alpha$ is a meridian boundary we will be writing $\left[D_{\alpha}\right]$ for the corresponding vertex in $\mathcal{D}\left(H_{g}\right)$. We will be saying that $\left[D_{\alpha}\right]$ is a meridian vertex and $\alpha$ a meridian curve. A vertex in $\mathcal{I}\left(H_{g}\right) \backslash\left(\mathcal{D}\left(H_{g}\right) \cup \mathcal{A}\left(H_{g}\right)\right)$ will be called a pants vertex.

By writing $[\alpha] \cap[\beta]=\varnothing$ for non-isotopic curves $\alpha, \beta$ we mean that there exist curves $\alpha^{\prime} \in[\alpha]$ and $\beta^{\prime} \in[\beta]$ such that $\alpha^{\prime} \cap \beta^{\prime}=\varnothing$. By writing $[\alpha] \cap[\beta] \neq \varnothing$ we mean that for any $\alpha^{\prime} \in[\alpha]$ and $\beta^{\prime} \in[\beta], \alpha^{\prime} \cap \beta^{\prime} \neq \varnothing$. By saying that the class $[\alpha]$ intersects the class $[\beta]$ at one point we mean that, in addition to $[\alpha] \cap[\beta] \neq \varnothing$, there exist curves $\alpha^{\prime} \in[a]$ and $\beta^{\prime} \in[\beta]$ which intersect at exactly one point.

The above notation with square brackets will be similarly used for surfaces. If $S$ is an incompressible surface we will denote by $L k([S])$ the link of the vertex $[S]$ in $\mathcal{I}\left(H_{g}\right)$, namely, for each simplex $\sigma$ containing $[S]$ consider the faces of $\sigma$ not containing $[S]$ and take the union over all such $\sigma$. We will use the notation $¥$ to declare that two links are not isomorphic as complexes.

We will also use the classical notation $\Sigma_{n, b}$ to denote a surface of genus $n$ with $b$ boundary components.

\section{Properties of the complex $\mathcal{I}\left(H_{g}\right)$}

In this section we will show that every automorphism of $\mathcal{I}(M)$ must preserve the subcomplexes $\mathcal{A}\left(H_{g}\right)$ and $\mathcal{D}\left(H_{g}\right)$. In particular, we will show that for $[S] \in$ $\mathcal{I}\left(H_{g}\right)$, the topological type of the surface $S$ determines the link of $[S]$ in $\mathcal{I}\left(H_{g}\right)$ and vice-versa. To do this we will find topological properties for the link of each 


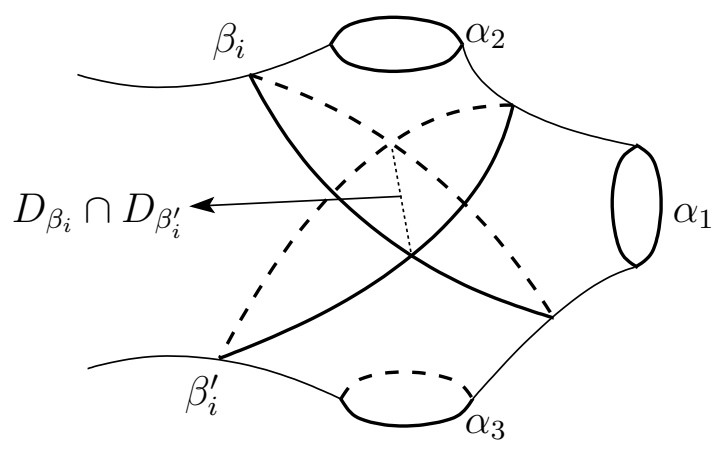

Figure 3:

topological type of surfaces (meridians, annuli and pairs of pants) that distinguish their links.

It is well known that a pants decomposition for $\partial H_{g}$ is a collection $\alpha_{1}, \ldots, \alpha_{3 g-3}$ of $3 g-3$ essential, non-parallel, simple closed curves such that the closure of each component of the complement of these curves is a pair of pants. The number of pairs of pants is $2 g-2$. Thus, the maximal number of vertices in a simplex of $\mathcal{I}\left(H_{g}\right)$ is $5 g-5$. In other words the dimension of $\mathcal{I}\left(H_{g}\right)$ is $\leq 5 g-6$. To see that simplices of dimension $5 g-6$ actually exist, observe that there exists a pants decomposition $\alpha_{1}, \ldots, \alpha_{3 g-3}$ so that each $\alpha_{i}$ is a non-separating, non-meridian curve for all $i$. This is displayed in Figure 1 for $g \geq 3$ and for $g=2$ see Remark 6 below. For such a choice of $\alpha_{i}$ 's, all $2 g-2$ pairs of pants formed by $\alpha_{1}, \ldots, \alpha_{3 g-3}$ are incompressible surfaces. Apparently, all such pairs of pants give rise to distinct elements in $\mathcal{I}\left(H_{g}\right)$. Thus, a pants decomposition $\alpha_{1}, \ldots, \alpha_{3 g-3}$ with all $\alpha_{i}$ 's being non-meridian curves gives rise to $3 g-3$ annular surfaces $S_{\alpha_{1}}, \ldots, S_{\alpha_{3 g-3}}$. These surfaces along with the $2 g-2$ pairs of pants formed by $\alpha_{1}, \ldots, \alpha_{3 g-3}$ give rise to a simplex in $\mathcal{I}\left(H_{g}\right)$ containing $5 g-5$ vertices. We have established the following

Proposition 2 The dimension of the complex $\mathcal{I}\left(H_{g}\right)$ is $5 g-6$.

We next examine the dimension of $L k([D])$ when $D$ is a meridian and of $L k\left(\left[S_{\alpha}\right]\right)$ when $S_{\alpha}$ is an annular surface.

Lemma 3 If $S_{\alpha}$ is an annular (incompressible) surface then the link of the vertex $\left[S_{\alpha}\right]$ in $\mathcal{I}\left(H_{g}\right)$ has dimension $5 g-7$.

Proof. We first assume that $\alpha$ is a separating curve. Then $\alpha$ decomposes $\partial H_{g}$ into surfaces $\Sigma_{n, 1}$ and $\Sigma_{m, 1}$ with $m+n=g$ and $m, n \geq 1$ with $\alpha$ being isotopic to the boundary of $\Sigma_{n, 1}$ as well as to the boundary of $\Sigma_{m, 1}$. To complete the proof in this case, it suffices to find a pants decomposition for $\partial H_{g}$ consisting 
of non-meridian curves and containing the curve $\alpha$. For the latter, it suffices to show the following

Claim $\Sigma_{n, 1}$ can be decomposed into $2 n-1$ pairs of pants so that the boundary curves of each are non-meridian when viewed as curves in $\partial H_{g}$.

The first step is to find pair-wise disjoint non-separating curves $\alpha_{1}, \ldots, \alpha_{n}$ in $\Sigma_{n, 1}$ such that $\alpha_{i}$ does not bound a disk in $H_{g}$ for all $i$. To see this, let $\alpha_{1}, \alpha_{1}^{\prime}$ be two simple non-separating curves in $\partial H_{g}$ such that the curves $\alpha, \alpha_{1}, \alpha_{1}^{\prime}$ bound a pair of pants in $\partial H_{g}$. As $\alpha$ is not the boundary of a meridian in $H_{g}$, it is clear that $\alpha_{1}, \alpha_{1}^{\prime}$ cannot both be meridian boundaries in $H_{g}$. Assuming $\alpha_{1}$ is not meridian boundary, we may cut $\Sigma_{n, 1}$ along $\alpha_{1}$ to obtain a surface $\Sigma_{n-1,3}$. By the same argument, we may find a non-separating curve $\alpha_{i}$ in $\Sigma_{n-(i-1), 2 i-1}, i=2, \ldots, n$ which is not meridian boundary.

Apparently, cutting $\Sigma_{n, 1}$ along $\alpha_{1}, \ldots, \alpha_{n}$ we obtain a sphere $\Sigma_{0,1+2 n}$ with $1+$ $2 n$ holes, such that the boundary components of $\Sigma_{0,1+2 n}$ do not bound disks when viewed as curves in $\partial H_{g}$. We now claim that we may find pair-wise disjoint curves $\beta_{1}, \ldots, \beta_{2 n-2}$ such that $\beta_{j}$ does not bound a disk in $H_{g}$ for all $j=1, \ldots, 2 n-2$. To see this, let $\beta_{1}, \beta_{1}^{\prime}$ be two simple closed curves in $\Sigma_{0,1+2 n}$ such that the curves $\alpha_{1}, \alpha_{2}, \beta_{1}$ bound a pair of pants and the curves $\alpha_{1}, \alpha_{3}, \beta_{1}^{\prime}$ bound a pair of pants as shown in Figure 3 . If both $\beta_{1}, \beta_{1}^{\prime}$ bound properly embedded disks in $H_{g}$, say $D_{\beta_{1}}, D_{\beta_{1}^{\prime}}$ respectively, then $D_{\beta_{1}} \cap D_{\beta_{1}^{\prime}}$ is a properly embedded arc in $H_{g}$ which separates $D_{\beta_{1}}$ into two half-disks. Similarly for $D_{\beta_{1}^{\prime}}$. Appropriate unions of these half-disks along $D_{\beta_{1}} \cap D_{\beta_{1}^{\prime}}$ establish a contradiction since none of $\alpha_{1}, \alpha_{2}, \alpha_{3}$ is a meridian boundary. Thus, at least one of $\beta_{1}, \beta_{1}^{\prime}$, say $\beta_{1}$, does not bound a disk. Cutting $\Sigma_{0,1+2 n}$ along $\beta_{1}$ we obtain a pair of pants and a surface $\Sigma_{0,1+2 n-1}$ which has the same property as $\Sigma_{0,1+2 n}$, namely, all boundary components of $\Sigma_{0,1+2 n-1}$ do not bound disks when viewed as curves in $\partial H_{g}$. By applying the same argument repeatedly, we may find the desired collection of curves $\beta_{1}, \ldots, \beta_{2 n-2}$ none of which is a meridian boundary. Apparently, the collection of curves $\beta_{1}, \ldots, \beta_{2 n-2}$ decomposes $\Sigma_{0,1+2 n}$ into $2 n-1$ pairs of pants as required. This completes the proof of the Claim and the proof of the lemma in the case $\alpha$ is separating.

Assume now that $\alpha$ is non-separating. Using two copies of $\alpha$ and a simple arc joining them we may construct a separating curve $\beta$ which decomposes $\partial H_{g}$ into surfaces $\Sigma_{g-1,1}$ and $\Sigma_{1,1}$ with $\beta$ being isotopic to the boundary of $\Sigma_{g-1,1}$ as well as to the boundary of $\Sigma_{1,1}$. Note that $\Sigma_{1,1}$ contains $\alpha$. Then by the above claim we have that $\Sigma_{g-1,1}$ can be decomposed into $2(g-1)-1$ (incompressible) pairs of pants by using non-meridian curves $\alpha_{i}, i=1, \ldots, 3 g-5$ contained in $\Sigma_{g-1,1}$ together with the cirve $\beta$. By adding the curve $\alpha$ we obtain a pants decomposition $\alpha_{1}, \ldots, \alpha_{3 g-5}, \beta, \alpha$ with all curves being non-meridian. Hence, $\left[S_{\alpha}\right]$ is contained in a simplex of maximum dimension, namely, of dimension $5 g-6$ which shows that the dimension of $L k\left(\left[S_{\alpha}\right]\right)$ is $5 g-7$. 
Lemma 4 If $D$ is a meridian then the link of the vertex $[D]$ in $\mathcal{I}\left(H_{g}\right)$ has dimension $5 g-9$.

Proof. First assume that $[D]$ is non-separating. We may find a pants decomposition $\alpha_{1}, \ldots, \alpha_{3 g-4}, \alpha_{3 g-3}=\partial D$ for $\partial H_{g}$ such that $\alpha_{i}$ is non-meridian for all $i=1, \ldots, 3 g-4$ (see Figure 21). This collection of curves decomposes $\partial H_{g}$ into $2 g-2$ pairs of pants such that exactly two of these have $\partial D$ as boundary component and, hence, they are compressible surfaces. Thus, a non-separating meridian $[D]$ is contained in a simplex with $3 g-3+2 g-4$ vertices and, hence, the dimension of $L k([D])$ is $\geq 5 g-9$. Let now $\alpha_{1}^{\prime}, \ldots, \alpha_{3 g-4}^{\prime}, \alpha_{3 g-3}=\partial D$ be any pants decomposition with corresponding pairs of pants $P_{1}, \ldots, P_{2 g-2}$ such that one of them, say $P_{1}$, has two boundary components isotopic to $\partial D$. Then the third boundary component of $P_{1}$ will also be a meridian, thus, another pair of pants distinct from $P_{1}$ will also be compressible. This shows that a class $[D]$ with $D$ non-separating meridian cannot be contained in a simplex of more that $5 g-7$ vertices and, thus, $L k([D])$ is equal to $5 g-9$.

If $[D]$ is separating, it is clear that any decomposition $\alpha_{1}, \ldots, \alpha_{3 g-4}, \alpha_{3 g-3}=$ $\partial D$ for $\partial H_{g}$ with $\alpha_{i}$ being non-meridian for all $i=1, \ldots, 3 g-4$ has the property that exactly two of the corresponding pairs of pants are compressible and we work similarly.

Proposition 5 Let $[D]$ be a meridian vertex, $\left[S_{\alpha}\right]$ an annular vertex and $[P]$ a pants vertex. Then the links $L k([D]), L k\left(\left[S_{\alpha}\right]\right)$ and $L k([P])$ are pair-wise nonisomorphic as complexes.

Proof. By the previous two Lemmata, the links of the vertices $[D]$ and $\left[S_{\alpha}\right]$ have distinct dimensions, hence, it is clear that $L k([D]) \nsucceq L k\left(\left[S_{\alpha}\right]\right)$. It remains to distinguish $L k([P])$ from $L k([D])$ and $L k\left(\left[S_{\alpha}\right]\right)$.

Let $[P]$ be a vertex in $\mathcal{I}(M)$ such that $P$ is a pair of pants with boundary components $\beta, \gamma, \delta$. The vertices in $L k([P])$ form a cone graph, that is, the vertex $\left[S_{\beta}\right]$ belongs to $L k([P])$ and is connected by an edge with any vertex in $L k([P])$. We will reach a contradiction by showing that

$$
\forall[Q] \in \operatorname{Lk}([D]), \exists[R] \in \operatorname{Lk}([D]):[Q] \cap[R] \neq \varnothing
$$

and similarly for $L k\left(\left[S_{\alpha}\right]\right)$. For, if $\beta_{Q}$ is a boundary component of a surface representing $[Q] \in L k([D])$ then there exists a curve $\gamma$ such that $\partial D \cap \gamma=\varnothing$ and $\gamma \cap \beta_{Q} \neq \varnothing$. Let $[R]$ be the vertex represented by $S_{\gamma}$ if $\gamma$ is non-meridian and by $D_{\gamma}$ if $\gamma$ is a meridian boundary. Then $[R] \in L k([D])$ is the required vertex which is not connected by an edge with $[Q]$, thus $L k([D])$ satisfies property $(*)$. Similarly, we show that $L k\left(\left[S_{\alpha}\right]\right)$ also satisfies property $(*)$.

Remark 6 Let $\alpha, \beta, \gamma$ be non-separating curves in $\partial \mathrm{H}_{2}$ decomposing $\partial \mathrm{H}_{2}$ into two components which we denote by $P, P^{\prime}$. Note that $P, P^{\prime}$ may not be isotopic. To 
see this, denote by $f_{1}, f_{2}$ the generators of $\pi_{1}\left(H_{2}\right)$ corresponding to the longitudes of $\mathrm{H}_{2}$. We may choose non-separating curves $\alpha, \beta$ on $\partial \mathrm{H}_{2}$ which represnt the second powers $f_{1}^{2}, f_{2}^{2}$ up to conjugacy. Choose an essential non-separating curve $\gamma$ such that $\alpha, \beta, \gamma$ are mutually disjoint and non isotopic. These curves separate $\partial \mathrm{H}_{2}$ into two components (pairs of pants) $P$ and $P^{\prime}$. If $P, P^{\prime}$ were isotopic then $\mathrm{H}_{2}$ would be homeomorphic to the product $P \times[0,1]$ and any two of the boundary components of $P$ would give rise to generators for $\pi_{1}\left(H_{2}\right)$. Since neither $\alpha \simeq f_{1}^{2}$ nor $\beta \simeq f_{2}^{2}$ are generators for the free group on $f_{1}, f_{2}$ it follows that, for this particular choice of $\alpha, \beta, \gamma$, the surfaces $P, P^{\prime}$ are not isotopic.

\section{Proof of the Main Theorem}

Let

$$
A: \mathcal{M C G}\left(H_{g}\right) \rightarrow \operatorname{Aut}\left(\mathcal{I}\left(H_{g}\right)\right)
$$

be the map sending a mapping class $F$ to the automorphism it induces on $\mathcal{I}\left(H_{g}\right)$, that is, $A(F)$ is given by

$$
A(F)[S]:=[F(S)]
$$

Theorem 7 The map $A: \mathcal{M C G}\left(H_{g}\right) \rightarrow \operatorname{Aut}\left(\mathcal{I}\left(H_{g}\right)\right)$ is onto for $g \geq 2$ and injective for $g \geq 3$. For $g=2$, A has a $\mathbb{Z}_{2}$-kernel generated by the hyper-elliptic involution.

We will use the following immediate Corollary of Proposition 5.

Corollary 8 Automorphisms of $\mathcal{I}\left(H_{g}\right)$ preserve all types (meridian, annular and pants) of vertices.

We will also need the following

Lemma 9 If $f \in \operatorname{Aut}\left(\mathcal{I}\left(H_{g}\right)\right)$ and $\left.f\right|_{\mathcal{D}\left(H_{g}\right) \cup \mathcal{A}\left(H_{g}\right)}=i d_{\mathcal{D}\left(H_{g}\right) \cup \mathcal{A}\left(H_{g}\right)}$ then $f([S])=$ $[S]$ for any vertex $[S] \in \mathcal{I}(M)$ except in the case mentioned in Remark 6 , namely, if $g=2$ and $P$ is a pair of pants with all boundary components of $\partial P$ being separating curves decomposing $\partial H_{2}$ into 2 components $P, P^{\prime}$, then either, $f([P])=[P]$ or, $f([P])=\left[P^{\prime}\right]$.

Proof. We have to show that $f \in \operatorname{Aut}\left(\mathcal{I}\left(H_{g}\right)\right)$ fixes every vertex $[P]$ where $P$ is a pair of pants. Let $[P]$ be such a vertex in $\mathcal{I}\left(H_{g}\right)$. By Corollary 8 it is clear that $f([P])$ is a vertex $\left[P^{\prime}\right]$ with $P^{\prime}$ being a pair of pants. Denote by $\alpha_{1}, \alpha_{2}, \alpha_{3}$ the boundary components of $P$ and, similarly, $\alpha_{1}^{\prime}, \alpha_{2}^{\prime}, \alpha_{3}^{\prime}$ for $P^{\prime}$. If $\left[\alpha_{i_{0}}\right] \cap\left[\alpha_{j_{0}}^{\prime}\right] \neq \varnothing$ for some $i_{0}, j_{0} \in\{1,2,3\}$ then the vertex $\left[S_{\alpha_{i_{0}}}\right]$ is connected by an edge with $[P]$ 
and is not connected by an edge with $\left[P^{\prime}\right]$. As $\left[S_{\alpha_{i_{0}}}\right]$ is fixed by $f$, it follows that $f([P])$ cannot be equal to $\left[P^{\prime}\right]$. Thus, we may assume that

$$
\left[\alpha_{i}\right] \cap\left[\alpha_{j}^{\prime}\right]=\varnothing \text { for all } i, j=1,2,3 .
$$

Consider the following property:

$$
\text { Up to change of enumeration, } \alpha_{i} \simeq \alpha_{i}^{\prime} \text { for } i=1,2,3 . \quad(* * *)
$$

If property $(* * *)$ holds then $P \simeq P^{\prime}$ unless $g=2$ and $\alpha_{1}, \alpha_{2}, \alpha_{3}$ are all non-separating curves which decompose $\partial \mathrm{H}_{2}$ into 2 pairs of pants (cf. Remark 6) which may or may not be isotopic. Thus, if property $(* * *)$ holds then either $f([P])=[P]$ or the exception in the statement of the lemma occurs.

We examine now the case where $g \geq 3$ and property $(* * *)$ does not hold. By assumption $(* *)$, we may cut $\partial H_{g}$ along $\alpha_{1}, \alpha_{2}, \alpha_{3}$ to obtain either

- the surface $P$ and a surface $\Sigma_{g-2,3}$ (if all $\alpha_{1}, \alpha_{2}, \alpha_{3}$ are non-separating) or,

- the surface $P$, a surface $\Sigma_{g_{1}, 1}$ and a surface $\Sigma_{g-g_{1}-1,2}$ for some $0<g_{1}<g$ (if exactly one of $\alpha_{1}, \alpha_{2}, \alpha_{3}$ is separating and the other two curves are non-isotopic) or,

- the surface $P$ and a surface $\Sigma_{g-1,1}$ (if exactly one of $\alpha_{1}, \alpha_{2}, \alpha_{3}$ is separating and the other two curves are isotopic) or,

- the surface $P$ and surfaces $\Sigma_{g_{1}, 1}, \Sigma_{g_{2}, 1}, \Sigma_{g_{3}, 1}$ for some $g_{1}, g_{2}, g_{3} \geq 1$ with $g_{1}+g_{2}+g_{3}=g$ (if all $\alpha_{1}, \alpha_{2}, \alpha_{3}$ are separating)

Note that if $P$ is a pair of pants, it is impossible to have exactly two of its boundary curves $\alpha_{1}, \alpha_{2}, \alpha_{3}$ being separating. In all cases, $P^{\prime}$ is contained in a surface of the form $\Sigma_{g^{\prime}, b}$ for some $g^{\prime} \in\{1, \ldots, g-1\}$ and $b \in\{1,2,3\}$ mentioned above. Thus, we may find a non-meridian curve $\alpha$ in $\partial H_{g}$ such that

$$
\alpha \cap \alpha_{i}=\varnothing, \forall i=1,2,3 \text { and }[\alpha] \cap\left[\alpha_{j_{0}}^{\prime}\right] \neq \varnothing \text { for some } j_{0} \in\{1,2,3\} .
$$

Then, for the annular surface $S_{\alpha}$ we have that $\left[S_{\alpha}\right]$ is connected by an edge with $[P]$ and is not connected by an edge with $\left[P^{\prime}\right]$. As $\left[S_{\alpha}\right]$ is fixed by $f$, it follows that $f([P])$ cannot be equal to $\left[P^{\prime}\right]$. This completes the proof of the lemma.

Proof of Theorem [7. We will use the corresponding result for surfaces, see [7], [9], which applies to the boundary of the handlebody $\partial H_{g}$.

We first show that every $f \in \operatorname{Aut}\left(\mathcal{I}\left(H_{g}\right)\right)$ is geometric. By Proposition 5 we know that $f\left(\mathcal{A}\left(H_{g}\right)\right)=\mathcal{A}\left(H_{g}\right)$ and $f\left(\mathcal{D}\left(H_{g}\right)\right)=\mathcal{D}\left(H_{g}\right)$. In particular, $f\left(\mathcal{C}\left(\partial H_{g}\right)\right)=\mathcal{C}\left(\partial H_{g}\right)$. The restriction $\left.f\right|_{\mathcal{C}\left(\partial H_{g}\right)}$ of $f$ on $\mathcal{C}\left(\partial H_{g}\right)$ induces an automorphism of $\mathcal{C}\left(\partial H_{g}\right)$ which by the analogous result for surfaces (see [7], [9]) is geometric, that is, there exists a homeomorphism

$$
F_{\partial H_{g}}: \partial H_{g} \rightarrow \partial H_{g}
$$


such that $A\left(F_{\partial H_{g}}\right)=\left.f\right|_{\mathcal{C}\left(\partial H_{g}\right)}$. As $\left.f\right|_{\mathcal{C}\left(\partial H_{g}\right)}$ maps $\mathcal{D}(M)$ to $\mathcal{D}(M), F_{\partial H_{g}}$ sends meridian boundaries to meridian boundaries. It follows that $F_{\partial H_{g}}$ extends to a homeomorphism $F: H_{g} \rightarrow H_{g}$. We know that $A(F)=f$ on $\mathcal{C}\left(\partial H_{g}\right)$ and we must show that $A(F)=f$ on $\mathcal{I}\left(H_{g}\right)$. This follows from Lemma 9 which completes the proof that every $f \in \operatorname{Aut}\left(\mathcal{I}\left(H_{g}\right)\right)$ is geometric.

Let $f \in \operatorname{Aut}\left(\mathcal{I}\left(H_{g}\right)\right)$. Since $A$ is shown to be onto, there exists a homeomorphism $F: H_{g} \rightarrow H_{g}$ such that $A([F])=f$. This implies that $f\left(\mathcal{D}\left(H_{g}\right)\right)=\mathcal{D}\left(H_{g}\right)$ and $f\left(\mathcal{A}\left(H_{g}\right)\right)=\mathcal{A}\left(H_{g}\right)$. In particular, $f$ restricted to $\mathcal{C}\left(\partial H_{g}\right) \equiv \mathcal{D}\left(H_{g}\right) \cup \mathcal{A}\left(H_{g}\right)$ induces an automorphism $\bar{f}$ of the complex of curves $\mathcal{C}\left(\partial H_{g}\right)$. By [7], 9], there exists a homeomorphism $F_{\partial H_{g}}: \partial H_{g} \rightarrow \partial H_{g}$ such that $A\left(F_{\partial H_{g}}\right)=\bar{f}$. Such a homeomorphism is unique unless $g=2$ in which case the map

$$
\mathcal{M C G}\left(\partial H_{2}\right) \rightarrow \operatorname{Aut}\left(\mathcal{C}\left(\partial H_{2}\right)\right)
$$

has a $\mathbb{Z}_{2}$-kernel generated by an involution of $\partial H_{2}$. However, any homeomorphism of $\partial H_{g}$ which extends to $H_{g}$ it does so uniquely (see, for example, [5, Theorem 3.7 p.94]), and therefore the map

$$
\mathcal{M C G}\left(H_{g}\right) \rightarrow \operatorname{Aut}\left(\mathcal{I}\left(H_{g}\right)\right)
$$

is injective unless $g=2$ in which case it has a $\mathbb{Z}_{2}$-kernel.

\section{Applications}

We first establish hyperbolicity for $\mathcal{I}\left(H_{g}\right)$.

Proposition 10 The complex $\mathcal{I}\left(H_{g}\right)$ is $\delta$-hyperbolic in the sense of Gromov.

Proof. As far as hyperbolicity is concerned, the 1-skeleton $\mathcal{I}\left(H_{g}\right)^{(1)}$ of $\mathcal{I}\left(H_{g}\right)$ is relevant. $\mathcal{I}\left(H_{g}\right)^{(1)}$ is endowed with the combinatorial metric so that each edge has length 1. Apparently, we have an embedding

$$
i: \mathcal{C}\left(\partial H_{g}\right)^{(1)} \hookrightarrow \mathcal{I}\left(H_{g}\right)^{(1)}
$$

with $i\left(\mathcal{C}\left(\partial H_{g}\right)^{(1)}\right)=\mathcal{D}\left(H_{g}\right)^{(1)} \cup \mathcal{A}\left(H_{g}\right)^{(1)}$ where the superscript ${ }^{(1)}$ always denotes 1 -skeleton. We claim that this embedding is isometric. Indeed, if $\left[\alpha_{1}\right],\left[\alpha_{2}\right]$ are distinct vertices with distance $d_{\mathcal{C}}\left(\left[\alpha_{1}\right],\left[\alpha_{2}\right]\right)$ in $\mathcal{C}\left(\partial H_{g}\right)^{(1)}$ then the distance $d_{\mathcal{I}}\left(i\left(\left[\alpha_{1}\right]\right), i\left(\left[\alpha_{2}\right]\right)\right)$ cannot be smaller. For, if $\left[S_{0}\right]=i\left(\left[\alpha_{1}\right]\right),\left[S_{1}\right], \ldots,\left[S_{k}\right]=$ $i\left(\left[\alpha_{2}\right]\right)$ is a sequence of vertices which gives rise to a geodesic in $\mathcal{I}(M)^{(1)}$ of length less than $d_{\mathcal{C}}\left(\left[\alpha_{1}\right],\left[\alpha_{2}\right]\right)$, equivalently,

$$
d_{\mathcal{I}}\left(i\left(\left[\alpha_{1}\right]\right), i\left(\left[\alpha_{2}\right]\right)\right)=k<d_{\mathcal{C}}\left(\left[\alpha_{1}\right],\left[\alpha_{2}\right]\right)
$$


then for each $j=1,2, \ldots, k-1$ consider $\beta_{j}$ to be any boundary component of $S_{j}$. It is clear that $\beta_{j}$ is disjoint from $\beta_{j-1}$ and $\beta_{j+1}$. Therefore, the sequence $\left[\alpha_{1}\right],\left[\beta_{1}\right], \ldots,\left[\beta_{k-1}\right],\left[\alpha_{2}\right]$ is a segment in $\mathcal{C}\left(\partial H_{g}\right)^{(1)}$ of length $k$ with $k<d_{\mathcal{C}}\left(\left[\alpha_{1}\right],\left[\alpha_{2}\right]\right)$, a contradiction.

For any vertex $[P]$ in $\mathcal{I}\left(H_{g}\right)^{(1)} \backslash \mathcal{D}\left(H_{g}\right)^{(1)} \cup \mathcal{A}\left(H_{g}\right)^{(1)}$ we may find an annular vertex, namely, $\left[S_{\partial P}\right]$ where $\partial P$ is any component of the boundary of $P$, which is connected by an edge with $[P]$. Thus, $\mathcal{I}\left(H_{g}\right)^{(1)}$ is within bounded distance from $i\left(\mathcal{C}\left(\partial H_{g}\right)^{(1)}\right)$. Since $\mathcal{C}\left(\partial H_{g}\right)^{(1)}$ is $\delta$-hyperbolic in the sense of Gromov, so is $\mathcal{I}\left(H_{g}\right)^{(1)}$.

An element $F \in \mathcal{M C G}\left(H_{g}\right)$ is called pseudo-Anosov when it restricts to a pseudo-Anosov homeomorphism on $\partial H_{g}$. The proof of the following proposition is immediate from the corresponding result for surfaces (see [10, Prop. 4.6]) along with the above mentioned fact that $\mathcal{C}\left(\partial H_{g}\right)$ is cobounded in $\mathcal{I}\left(H_{g}\right)$.

Proposition 11 For any $g \geq 2$, there exists a $c>0$ such that any pseudo-Anosov $F \in \mathcal{M C G}\left(H_{g}\right)$, any vertex $v \in \mathcal{I}\left(H_{g}\right)$ and any $n \in \mathbb{Z}$,

$$
d_{\mathcal{I}}\left(F^{n}(v), v\right) \geq c|n| .
$$

Thus, pseudo-Anosov elements in $\mathcal{M C G}\left(H_{g}\right)$ correspond to hyperbolic isometries of $\mathcal{I}\left(H_{g}\right)$ and there are no parabolic isometries for $\mathcal{I}\left(H_{g}\right)$.

\section{References}

[1] E. Akbas, A presentation for the automorphisms of the 3-sphere that preserve a genus two Heegaard splitting, Pacific J. Math. 236 (2008), no. 2, 201-222.

[2] B.H. Bowditch, Intersection numbers and the hyperbolicity of the curve complex, J. Reine Angew. Math. 598 (2006), pp. 105-129.

[3] Ch. Charitos, I. Papadoperakis, G. Tsapogas, Incompressible surfaces in handlebodies and isotopies, Topology and its Applications, Volume 155, Issue 7 (2008), pp. 696-724.

[4] S. Cho, Homeomorphisms of the 3-sphere that preserve a Heegaard splitting of genus two, Proc. Amer. Math. Soc. 136 (2008), no. 3, 1113-1123.

[5] A.T. Fomenko, S.V. Matveev, Algorithmic and Computer Methods in 3manifolds, Kluwer Academic Publishers 1997.

[6] W. Harvey, Boundary structure of the modular group, Riemann surfaces and related topics, in: Proceedings of the 1978 Stony Brook Conference, State Univ. New York, Stony Brook, N.Y., 1978, Ann. Math. Stud. 97, Princeton Univ. Press, Princeton, N.J., 1981, pp. 245-251. 
[7] N.V. Ivanov, Automorphisms of complexes of curves and of Teichmuller spaces, Int. Math. Res. Notice 14 (1997) 651-666.

[8] M. Korkmaz, S. Schleimer, Automorphisms of the disk complex, http://arxiv.org/abs/0910.2038v1

[9] F. Luo, Automorphisms of the complex of curves, Topology 39 (2000) pp. 283-298.

[10] H. Masur, Y.N. Minsky, Geometry of the complex of curves. I. Hyperbolicity, Invent. Math. 138 (1999), no. 1, pp. 103-149.

[11] H. Masur, Y.N. Minsky, Quasiconvexity in the curve complex, In the tradition of Ahlfors and Bers, III, 309-320, Contemp. Math., 355, Amer. Math. Soc., Providence, RI, 2004.

[12] D. McCullough, Virtually geometrically finite mapping class groups of 3manifolds, J. Differential Geom. 33 (1991), no. 1, pp. 1-65.

[13] R. Qiu, Incompressible surfaces in handlebodies and closed 3-manifolds of Heegaard genus 2, Proc. of AMS, 128 No 10 (2000), pp. 3091-3097.

[14] M. Scharlemann, Automorphisms of the 3-sphere that preserve a genus two Heegaard splitting, Bol. Soc. Mat. Mexicana (3) 10 (2004), Special Issue, 503-514. 